\title{
EFFECT OF INSTANTANEOUS MASS EJECTION ON ORBITAL ELEMENTS OF CIRCULAR BINARIES
}

\author{
R. M I T A L A S
}

Dept. of Astronomy, University of Western Ontario, London, Canada

\begin{abstract}
All attempts to fit the binary pulsar PSR $1913+16$ into an evolutionary scenario consider the effect of an instantaneous mass ejection on the orbital elements of a circular binary.

In an explosion which does not affect the companion and does not change the linear velocity of the remnant, the original mass ratio of the binary is related to the final mass ratio by
\end{abstract}

$$
\frac{m_{1}}{m_{2}}=\left(\frac{q m_{1}}{m_{2}}\right)(1+e)+e
$$

where $q m_{1}$ is the mass remaining after the explosion of $m_{1}, m_{2}$ is the mass of the companion, and $e$ is the final eccentricity. There is an upper limit to the value of $q$, and a minimum to the fraction of mass carried away by the explosion,

$$
q_{\max }=\frac{1}{1+e} ; \quad f_{\min }=\frac{e}{1+e} .
$$

The total mass, period, semi-major axis and mass function of the original circular binary are simply related to the total mass, period, semi-major axis and mass function of the present binary. From the mass limits of pulsars, bounds can be set on the original mass of the exploding star,

$$
M_{p}(1+e)<m_{1}<M_{p} \frac{(1+e)}{(1-e)}
$$

where $M_{p}$ is the pulsar mass, and the right side inequality is valid only if the primary explodes. A lower limit on the original mass ratio is set, $\left(m_{1} / m_{2}\right) \geqslant e$.

An asymmetric, instantaneous explosion where both the mass and velocity of each component of the binary are altered also has an analytic solution. If the radial velocity increments of the remnants $q_{1} m_{1}$ and $q_{2} m_{2}$ due to the explosion are $f_{1} v$ and $f_{2} v$ where $v$ is the relative velocity, and $f_{3} v$ is the velocity increment of the exploding star $m_{1}$ in the direction opposite to its orbital velocity, then the total masses before and after the explosion are related by

$$
\left(m_{1}+m_{2}\right)=\left(q_{1} m_{1}+q_{2} m_{2}\right)\left[\frac{1 \pm e \sqrt{R}}{\mathrm{D}}\right]
$$

where $R=1-\frac{1-e^{2}}{e^{2}} \frac{\left(f_{1}+f_{2}\right)^{2}}{\left(1-f_{3}\right)^{2}}$ and $D=\left(f_{1}+f_{2}\right)^{2}+\left(1-f_{3}\right)^{2}$.

The permitted values of $\left(f_{1}+f_{2}\right), f_{3}$ are limited, since $R$ must be positive. For applications to the binary pulsar, limitation to $\left(q_{1} m_{1}+q_{2} m_{2}\right) /\left(m_{1}+m_{2}\right) \leqslant 1$ limits the allowed values of parameters further. 
The period, semi-major axis and mass function of the original circular binary are simply related to the period, semi-major axis and mass function of the present binary. It is noteworthy that the ratio of the semi-major axes is independent of $f_{3}$ when $\left(f_{1}+f_{2}\right)=0$. The maximum increase of the ratio of semi-major axes is $1 /(1-e)$. A full account will appear in a forthcoming publication.

Note Added in Proof. It has been pointed out to the author by Dr E. H. Geyer that the first equation is the same as the one found in Borsche, D.: 1962, Z. Astrophys. 56, 181. 